\title{
On Crossed Squares of Commutative Algebras
}

\author{
Elis Soylu Yılmaz* and Koray Yılmaz
}

\begin{abstract}
In this work, we show a categorical property for crossed squares of commutative algebras by defining a specific object in this category and then we give the construction of the pullback with this object.
\end{abstract}

Keywords: Crossed module; Crossed square; Pullback.

AMS Subject Classification (2020): Primary: 18 C40 ; Secondary: 18G45; 18C10; $18 D 30$

${ }^{*}$ Corresponding author

\section{Introduction}

Whitehead introduced crossed modules [1] as a homotopy 2-type connected space. In his work Whitehead shows that for a CW-complex

$$
\partial: \pi_{2}(X, P, *) \rightarrow \pi_{1}(P, *)
$$

is a crossed module. Later Porter adapts this notion for crossed modules of algebras [8]. The commutative algebra case for crossed modules can be found in the works of Gerstenhaber [9] and Lichtenbaum-Schlessinger [10].

Guin- Waléry and Loday defined crossed squares in [11] as an algebraic model for homotopy 3-type connected spaces. Thus crossed squares model homotopy types in dimensions bigger than 3. Later Ellis defined the commutative algebra version of crossed squares in [2]. 2- crossed modules and quadratic modules are also models for homotopy 3- types. Quadratic modules defined by Baues and 2-crossed modules defined by Conduché. The commutative algebra versions of quadratic modules and 2-crossed modules defined in [13], [14] respectively. Also Conduché shows that a mapping cone of a crossed square is a 2-crossed module [12]. The relations between the category of crossed squares and related categories such as simplicial groups, 2-crossed modules are given in [3], [4] and [13] as illustrated in the following diagram.

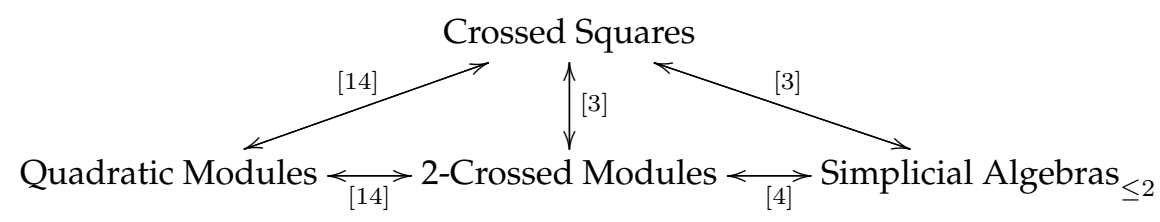

Categorical properties of these related categories such as pullback, limit and colimit are investigated in [5] [6], [7].The pullback construction is highly related with fibration. The fibration of 2-crossed modules is given in [16]. The category of pairs of crossed modules is mentioned in [7] to investigate the bifibration of crossed squares of groups. In section 3 we adapt this category for commutative algebras and construct a crossed squares from any pair of crossed modules. In last section, we construct the pullback of crossed squares over commutative algebras.

\section{Preliminaries}

In this section, we will recall the definitions of crossed modules and crossed squares. Now we give the definition of crossed modules of algebras from [15].

Received : 12-04-2020, Accepted : 01-08-2020 
Definition 2.1. Let $C$ and $R$ be two $k$-algebras and $R$ acts on $C$. The morphism

$$
\delta: C \rightarrow R
$$

of $k$-algebras is called pre crossed module if

$$
\delta(r \cdot c)=r \delta(c)
$$

for all $c$ in $C$ and $r$ in $R$. In addition to this condition if $\partial: C \rightarrow R$ satisfy

$$
c \cdot \delta\left(c^{\prime}\right)=c c^{\prime}
$$

then $\partial: C \rightarrow R$ is called a crossed module and denoted with $(C, R, \delta)$.

Example 2.2. Let $R$ be an algebra and $I$ be an ideal of $G$. $(I, R$, inc $)$ with inc : $I \hookrightarrow R$ is a crossed module. Where $R$ acts on $I$ by conjugation.

Example 2.3. Let $M$ be a $R$ - module. $\left(M, R, 0_{M}\right)$ is a crossed module with zero morphism $0_{M}: M \longrightarrow R$, $0_{M}(m)=e_{R}$.

Definition 2.4. A commutative diagram

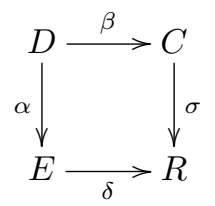

of commutative algebras with actions of the algebra $R$ on $C, D$ and $E$ and $h$-map

$$
h: C \times E \rightarrow D
$$

is called crossed square [2] if for all $c, c^{\prime} \in C, d \in D$ and $e, e^{\prime} \in E, r \in R, k \in \mathbf{k}$ :

1. The $\beta, \alpha, \sigma, \delta$ and $\sigma \beta=\delta \alpha$ are crossed modules;

2. $\quad \beta, \alpha$ preserve the action of $R$;

3. $\quad k h(c, e)=h(k c, e)=h(c, k e)$

4. $\quad h\left(c, e+e^{\prime}\right)=h(c, e)+h\left(c, e^{\prime}\right)$

5. $\quad h\left(c+c^{\prime}, e\right)=h(c, e)+h\left(c^{\prime}, e\right)$;

6. $\quad r \cdot h(c, e)=h(r \cdot c, e)=h(c, r \cdot e) ;$

7. $\quad \beta h(c, e)=c \cdot e$;

8. $\quad \alpha h(c, e)=e \cdot c$;

9. $\quad h(c, \alpha(d))=c \cdot d$

10. $\quad h(\beta(d), e)=e \cdot d$.

We will denote such a crossed square with $\left(\begin{array}{ccc} & C & \\ D & & R \\ & E & \end{array}\right)$. Let

$$
\varphi:\left(\begin{array}{ccc} 
& C & \\
D & & R \\
& E &
\end{array}\right) \rightarrow\left(\begin{array}{ccc} 
& C^{\prime} & \\
D^{\prime} & & R^{\prime} \\
& E^{\prime} &
\end{array}\right)
$$

be a morphism of crossed squares. Then the morphisms

$$
\begin{aligned}
& \varphi_{D}: D \longrightarrow D^{\prime} \quad \varphi_{C}: C \longrightarrow C^{\prime} \\
& \varphi_{E}: E \longrightarrow E^{\prime} \quad \varphi_{R}: R \longrightarrow R^{\prime}
\end{aligned}
$$


are crossed module morphisms making the diagram

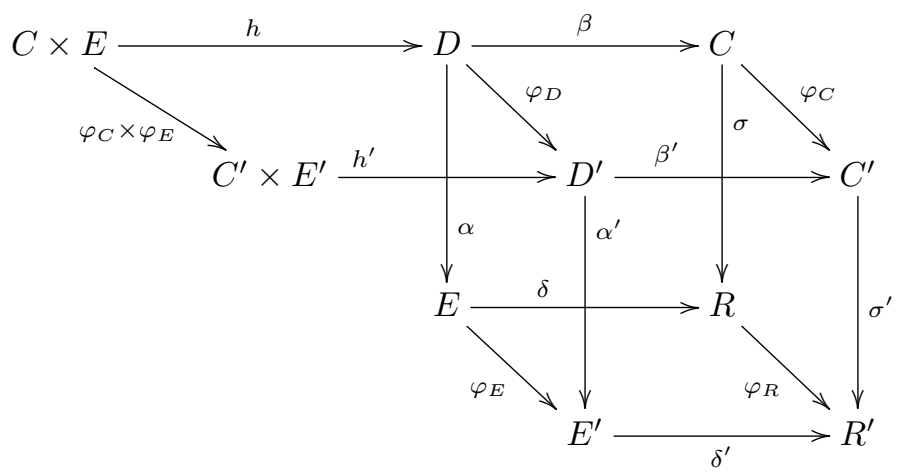

is commutative and the homomorphisms $\varphi_{D}, \varphi_{C}, \varphi_{E}$ are $\varphi_{R}$-equivariant. We will denote this category by $\mathrm{Crs}^{2}$.

Example 2.5. Let $(C, R, \partial)$ be a crossed modules over commutative algebras and $\left(C^{\prime}, R^{\prime}, \partial^{\prime}\right)$ be any ideal of $(C, R, \partial)$. The diagram

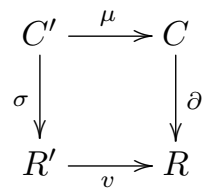

with $h: C \times R^{\prime} \rightarrow C^{\prime}$ h-map given by $h\left(c r^{\prime}\right)=c r^{\prime}$ for $c$ in $C$ and $r^{\prime}$ in $R^{\prime}$ is a crossed square.

Example 2.6. Let $I$ and $J$ be two ideals of a commutative ring $R$. The diagram

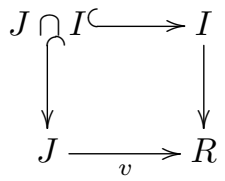

is a crossed square with $h: J \times I \rightarrow J \cap I$ and h-map is given by $h(j i)=j i$.

\section{Crossed Squares from Pairs of Crossed Modules}

In [7] Brown and Sivera mentioned bifibration of crossed squares over the category of pairs of crossed modules in groups. In this section, we will give the definition of the category, pairs of crossed modules, for commutative algebras.

Definition 3.1. Let $\mu: C \rightarrow P$ and $\vartheta: E \rightarrow P$ be crossed modules of commutative algebras. The category, pairs of crossed modules, $X M o d^{2}$ consists of objects

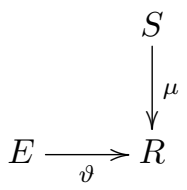

and with the morphisms preserving the action of $R$ on $C$ and $E$. Shortly we will write $(C, E, R, \mu, \vartheta)$ for a pair of crossed modules.

Let $\left(\begin{array}{ccc} & C & \\ D & & R \\ & E & \end{array}\right)$ be a crossed square and $f=\left(f_{1}, f_{2}, f_{3}\right):\left(C^{\prime}, E^{\prime}, R^{\prime}, \mu^{\prime}, \vartheta^{\prime}\right) \rightarrow(C, E, R, \mu, \vartheta)$ be a morphism 
in $X M o d^{2}$ as given by the following diagram.

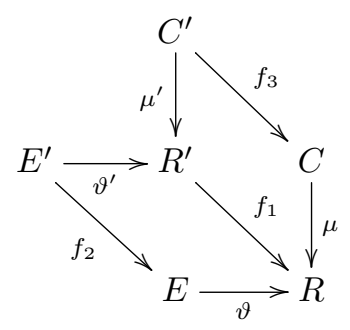

We define

$$
f^{*}=\left\{\left(e^{\prime}, c^{\prime}, d\right) \in E^{\prime} \times C^{\prime} \times D: \vartheta^{\prime}\left(e^{\prime}\right)=\mu^{\prime}\left(c^{\prime}\right), f_{2}\left(e^{\prime}\right)=\alpha(d), f_{3}\left(c^{\prime}\right)=\beta(d)\right\}
$$

and $\beta_{1}\left(e^{\prime}, c^{\prime}, d\right)=c^{\prime}, \beta_{2}\left(e^{\prime}, c^{\prime}, d\right)=e^{\prime}$ to give the next proposition where $\sigma: D \rightarrow C$ and $\delta: D \rightarrow E$.

Proposition 3.2. The diagram

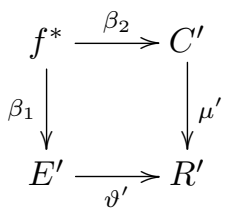

constructed with the data above is an object in $\mathrm{Crs}^{2}$.

Proof. 1. From the definition $\mu^{\prime}$ and $\vartheta^{\prime}$ are crossed modules. First, let us show that $\beta_{1}$ is a crossed module.

$$
\begin{aligned}
\beta_{1}\left(c^{\prime \prime} \cdot\left(e^{\prime}, c^{\prime}, d\right)\right) & =\beta_{1}\left(\mu^{\prime}\left(c^{\prime \prime}\right) \cdot e^{\prime}, c^{\prime \prime} \cdot c^{\prime}, f_{3}\left(c^{\prime \prime}\right) \cdot d\right) \\
& =c^{\prime \prime} \cdot c^{\prime} \\
& =c^{\prime \prime} \cdot \beta_{1}\left(e^{\prime}, c^{\prime}, d\right)
\end{aligned}
$$

for $c^{\prime \prime} \in C^{\prime},\left(e^{\prime}, c^{\prime}, d\right) \in f^{*}$ and

$$
\begin{aligned}
\left(\beta_{1}\left(e^{\prime}, c^{\prime}, d\right)\right) \cdot\left(e^{\prime}, c^{\prime \prime}, d^{\prime}\right) & =c^{\prime} \cdot\left(e^{\prime \prime}, c^{\prime \prime}, d^{\prime}\right) \\
& =\left(\mu^{\prime}\left(c^{\prime}\right) \cdot e^{\prime \prime}, c^{\prime} \cdot c^{\prime \prime}, f_{3}\left(c^{\prime \prime}\right) \cdot d\right) \\
& =\left(\vartheta^{\prime}\left(e^{\prime}\right) \cdot e^{\prime \prime}, c^{\prime} c^{\prime}, \sigma(d) \cdot d^{\prime}\right) \\
& =\left(e^{\prime} e^{\prime \prime}, c^{\prime} c^{\prime \prime}, d d^{\prime}\right) \\
& =\left(e^{\prime}, c^{\prime}, d\right)\left(e^{\prime \prime}, c^{\prime \prime}, d^{\prime}\right)
\end{aligned}
$$

for $\left(e^{\prime}, c^{\prime}, d\right),\left(e^{\prime \prime}, c^{\prime \prime}, d^{\prime}\right) \in f^{*}$. Similiar way $\beta_{2}$ is a crossed module. Since compositions of two crossed modules $\mu^{\prime} \beta_{1}, \vartheta^{\prime} \beta_{2}$ are crossed modules and from the definition of $f^{*}$ it is clear that $\mu^{\prime} \beta_{1}=\vartheta^{\prime} \beta_{2}$.

2. $\beta_{1}$ and $\beta_{2}$ preserves the action for $r^{\prime} \in R^{\prime}$ and $\left(e^{\prime}, c^{\prime}, d\right) \in f^{*}$

$$
\begin{aligned}
\beta_{2}\left(r^{\prime} \cdot\left(e^{\prime}, c^{\prime}, d\right)\right) & =\beta_{2}\left(r^{\prime} \cdot e^{\prime}, r^{\prime} \cdot c^{\prime}, f_{1}\left(r^{\prime}\right) \cdot d\right) \\
& =r^{\prime} \cdot e^{\prime} \\
& =r^{\prime} \cdot \beta_{2}\left(e^{\prime}, c^{\prime}, d\right)
\end{aligned}
$$

3. Define

$$
\begin{array}{ll}
h^{\prime}: E^{\prime} \times C^{\prime} & \rightarrow E^{\prime} \times C^{\prime} \times D \\
\left(e^{\prime}, c^{\prime}\right) & \mapsto\left(\mu^{\prime}\left(c^{\prime}\right) \cdot e^{\prime}, \vartheta^{\prime}\left(e^{\prime}\right) \cdot c^{\prime \prime}, h\left(f_{2}\left(e^{\prime}\right), f_{3}^{\prime}\left(c^{\prime \prime}\right)\right)\right)
\end{array}
$$

where $h: E \times C \rightarrow D$ is the h-map of $\left(\begin{array}{ccc} & C & \\ D & & R \\ & E & \end{array}\right)$. For $c^{\prime} \in C^{\prime}, e^{\prime} \in E^{\prime}$ and $k \in k$ we have

$$
\begin{aligned}
k \cdot h^{\prime}\left(e^{\prime}, c^{\prime}\right) & =k\left(\mu^{\prime}\left(c^{\prime}\right) \cdot e^{\prime}, \vartheta^{\prime}\left(e^{\prime}\right) \cdot c^{\prime \prime}, h\left(f_{2}\left(e^{\prime}\right), f_{3}^{\prime}\left(c^{\prime \prime}\right)\right)\right) \\
& =\left(k \cdot \mu^{\prime}\left(c^{\prime}\right) \cdot e^{\prime}, k \cdot \vartheta^{\prime}\left(e^{\prime}\right) \cdot c^{\prime \prime}, k \cdot h\left(f_{2}\left(e^{\prime}\right), f_{3}^{\prime}\left(c^{\prime \prime}\right)\right)\right) \\
& =\left(\mu^{\prime}\left(c^{\prime}\right) \cdot k e^{\prime}, \vartheta^{\prime}\left(k e^{\prime}\right) \cdot c^{\prime \prime}, h\left(k f_{2}\left(e^{\prime}\right), f_{3}^{\prime}\left(c^{\prime \prime}\right)\right)\right) \\
& =\left(\mu^{\prime}\left(c^{\prime}\right) \cdot k e^{\prime}, \vartheta^{\prime}\left(k e^{\prime}\right) c^{\prime \prime}, h\left(f_{2}\left(k e^{\prime}\right), f_{3}^{\prime}\left(c^{\prime \prime}\right)\right)\right) \\
& =h^{\prime}\left(k e^{\prime}, c^{\prime}\right)
\end{aligned}
$$




$$
\begin{aligned}
k \cdot h^{\prime}\left(e^{\prime}, c^{\prime}\right) & =k \cdot\left(\mu^{\prime}\left(c^{\prime}\right) \cdot e^{\prime}, \vartheta^{\prime}\left(e^{\prime}\right) \cdot c^{\prime \prime}, h\left(f_{2}\left(e^{\prime}\right), f_{3}^{\prime}\left(c^{\prime \prime}\right)\right)\right) \\
& =\left(k \cdot \mu^{\prime}\left(c^{\prime}\right) \cdot e^{\prime}, k \cdot \vartheta^{\prime}\left(e^{\prime}\right) \cdot c^{\prime \prime}, k \cdot h\left(f_{2}\left(e^{\prime}\right), f_{3}^{\prime}\left(c^{\prime \prime}\right)\right)\right) \\
& =\left(\mu^{\prime}\left(k c^{\prime}\right) \cdot e^{\prime}, \vartheta^{\prime}\left(e^{\prime}\right) \cdot k c^{\prime \prime}, h\left(f_{2}\left(e^{\prime}\right), k f_{3}^{\prime}\left(c^{\prime \prime}\right)\right)\right) \\
& =\left(\mu^{\prime}\left(k c^{\prime}\right) \cdot e^{\prime}, \vartheta^{\prime}\left(e^{\prime}\right) \cdot k c^{\prime \prime}, h\left(f_{2}\left(e^{\prime}\right), f_{3}^{\prime}\left(k c^{\prime \prime}\right)\right)\right) \\
& =h^{\prime}\left(e^{\prime}, k c^{\prime}\right)
\end{aligned}
$$

4. For $c^{\prime}, c^{\prime \prime} \in C^{\prime}$ and $e^{\prime} \in E^{\prime}$;

$$
\begin{aligned}
h^{\prime}\left(e^{\prime}, c^{\prime}+c^{\prime \prime}\right) & =\left(\mu^{\prime}\left(c^{\prime}+c^{\prime \prime}\right) \cdot e^{\prime}, \vartheta^{\prime}\left(e^{\prime}\right) \cdot\left(c^{\prime}+c^{\prime \prime}\right), h\left(f_{2}\left(e^{\prime}\right), f_{3}^{\prime}\left(c^{\prime}+c^{\prime \prime}\right)\right)\right) \\
& =\left(\left(\mu^{\prime}\left(c^{\prime}\right) \cdot e^{\prime}+\mu^{\prime}\left(c^{\prime \prime}\right) \cdot e^{\prime}, \vartheta^{\prime}\left(e^{\prime}\right) \cdot c^{\prime}+\vartheta^{\prime}\left(e^{\prime}\right) \cdot c^{\prime \prime}, h\left(f_{2}\left(e^{\prime}\right), f_{3}^{\prime}\left(c^{\prime}\right)+f_{3}^{\prime}\left(c^{\prime \prime}\right)\right)\right)\right. \\
& \left.=\left(\mu^{\prime}\left(c^{\prime}\right) \cdot e^{\prime}+\mu^{\prime}\left(c^{\prime \prime}\right) \cdot e^{\prime}, \mu^{\prime}\left(c^{\prime}\right) \cdot e^{\prime}\right)\right)+\vartheta^{\prime}\left(e^{\prime \prime \prime}, h\left(\left(f_{2}\left(e^{\prime}\right), f_{3}^{\prime}\left(c^{\prime}\right)\right), h\left(f_{2}\right)\left(e^{\prime}\right) f_{3}^{\prime}\left(c^{\prime \prime}\right)\right)\right. \\
& =\left(\mu^{\prime}\left(c^{\prime}\right) \cdot e^{\prime}, \mu^{\prime}\left(c^{\prime}\right) \cdot e^{\prime}, h\left(f_{2}\left(e^{\prime}\right), f_{3}^{\prime}\left(c^{\prime}\right)\right)\right)+\left(\mu^{\prime}\left(c^{\prime \prime}\right) \cdot e^{\prime}, \vartheta^{\prime}\left(e^{\prime}\right) \cdot c^{\prime \prime}, h\left(f_{2}\left(e^{\prime}\right) f_{3}^{\prime}\left(c^{\prime \prime}\right)\right)\right) \\
& =h^{\prime}\left(e^{\prime}, c^{\prime}\right)+h^{\prime}\left(e^{\prime}, c^{\prime \prime}\right)
\end{aligned}
$$

5.For $c^{\prime} \in C^{\prime}$ and $e^{\prime}, e^{\prime \prime} \in E^{\prime}$;

$$
\begin{aligned}
h^{\prime}\left(e^{\prime}+e^{\prime \prime}, c^{\prime}\right) & =\left(\mu^{\prime}\left(c^{\prime}\right) \cdot\left(e^{\prime}+e^{\prime \prime}\right), \vartheta^{\prime}\left(e^{\prime}+e^{\prime \prime}\right) \cdot c^{\prime}, h\left(f_{2}\left(e^{\prime}+e^{\prime \prime}\right), f_{3}^{\prime}\left(c^{\prime}\right)\right)\right) \\
& =\left(\mu^{\prime}\left(c^{\prime}\right) \cdot e^{\prime}+\mu^{\prime}\left(c^{\prime}\right) \cdot e^{\prime \prime},\left(\vartheta^{\prime}\left(e^{\prime}\right)+\vartheta^{\prime}\left(e^{\prime \prime}\right)\right) \cdot c^{\prime}, h\left(f_{2}\left(e^{\prime}\right)+f_{2}\left(e^{\prime \prime}\right),, f_{3}^{\prime}\left(c^{\prime}\right)\right)\right) \\
& =\left(\mu^{\prime}\left(c^{\prime}\right) \cdot e^{\prime}+\mu^{\prime}\left(c^{\prime}\right) \cdot e^{\prime \prime}, \vartheta^{\prime}\left(e^{\prime}\right) \cdot c^{\prime}+\vartheta^{\prime}\left(e^{\prime \prime}\right) \cdot c^{\prime}, h\left(\left(f_{2}\left(e^{\prime}\right), f_{3}^{\prime}\left(c^{\prime}\right)\right)+h\left(\left(f_{2}\left(e^{\prime \prime}\right), f_{3}^{\prime}\left(c^{\prime}\right)\right)\right)\right.\right. \\
& =\left(\mu^{\prime}\left(c^{\prime}\right) \cdot e^{\prime}, \vartheta^{\prime}\left(e^{\prime}\right) \cdot c^{\prime}, h\left(\left(f_{2}\left(e^{\prime}\right), f_{3}^{\prime}\left(c^{\prime}\right)\right)\right)+\left(\mu^{\prime}\left(c^{\prime}\right) \cdot e^{\prime \prime}, \vartheta^{\prime}\left(e^{\prime \prime}\right) \cdot c^{\prime}, h\left(\left(f_{2}\left(e^{\prime \prime}\right), f_{3}^{\prime}\left(c^{\prime}\right)\right)\right)\right.\right. \\
& =h^{\prime}\left(e^{\prime}, c^{\prime}\right)+h^{\prime}\left(e^{\prime \prime}, c^{\prime}\right)
\end{aligned}
$$

6. For $c^{\prime} \in C^{\prime}, e^{\prime}, \in E^{\prime}$ and $r^{\prime} \in R^{\prime}$;

$$
\begin{aligned}
r^{\prime} \cdot h^{\prime}\left(e^{\prime}, c^{\prime}\right) & =r^{\prime} \cdot\left(\mu^{\prime}\left(c^{\prime}\right) \cdot e^{\prime}, \vartheta^{\prime}\left(e^{\prime}\right) \cdot c^{\prime \prime}, h\left(f_{2}\left(e^{\prime}\right), f_{3}^{\prime}\left(c^{\prime}\right)\right)\right) \\
& =r^{\prime} \cdot\left(\mu^{\prime}\left(c^{\prime}\right) \cdot e^{\prime}\right), r^{\prime} \cdot\left(\vartheta^{\prime}\left(e^{\prime}\right) \cdot c^{\prime \prime}\right), r^{\prime} \cdot h\left(f_{2}\left(e^{\prime}\right), f_{3}^{\prime}\left(c^{\prime}\right)\right) \\
& =\left(r^{\prime} \cdot \mu^{\prime}\left(c^{\prime}\right)\right) \cdot e^{\prime},\left(r^{\prime} \cdot \vartheta^{\prime}\left(e^{\prime}\right)\right) \cdot c^{\prime \prime}, h\left(r^{\prime} \cdot f_{2}\left(e^{\prime}\right), f_{3}^{\prime}\left(c^{\prime}\right)\right) \\
& =\left(\mu^{\prime}\left(c^{\prime}\right) \cdot r^{\prime} \cdot e^{\prime}, \vartheta^{\prime}\left(r^{\prime} e^{\prime}\right) \cdot c^{\prime \prime}, h\left(f_{2}\left(r^{\prime} \cdot e^{\prime}\right), f_{3}^{\prime}\left(c^{\prime}\right)\right)\right) \\
& =\left(\mu^{\prime}\left(c^{\prime}\right) \cdot\left(r^{\prime} \cdot e^{\prime}\right), \vartheta^{\prime}\left(r^{\prime} \cdot e^{\prime}\right) \cdot c^{\prime \prime}, h\left(f_{2}\left(r^{\prime} \cdot e^{\prime}\right), f_{3}^{\prime}\left(c^{\prime}\right)\right)\right) \\
& =h^{\prime}\left(r^{\prime} \cdot e^{\prime}, c^{\prime}\right) \\
r^{\prime} \cdot h^{\prime}\left(e^{\prime}, c^{\prime}\right) & =r^{\prime} \cdot\left(\mu^{\prime}\left(c^{\prime}\right) \cdot e^{\prime}, \vartheta^{\prime}\left(e^{\prime}\right) \cdot c^{\prime \prime}, h\left(f_{2}\left(e^{\prime}\right), f_{3}^{\prime}\left(c^{\prime}\right)\right)\right) \\
& =r^{\prime} \cdot\left(\mu^{\prime}\left(c^{\prime}\right) \cdot e^{\prime}\right), r^{\prime} \cdot\left(\vartheta^{\prime}\left(e^{\prime}\right) \cdot c^{\prime \prime}\right), r^{\prime} \cdot h\left(f_{2}\left(e^{\prime}\right), f_{3}^{\prime}\left(c^{\prime}\right)\right) \\
& =\left(r^{\prime} \cdot \mu^{\prime}\left(c^{\prime}\right)\right) \cdot e^{\prime},\left(r^{\prime} \cdot \vartheta^{\prime}\left(e^{\prime}\right)\right) \cdot c^{\prime \prime}, h\left(r^{\prime} \cdot f_{2}\left(e^{\prime}\right), f_{3}^{\prime}\left(c^{\prime}\right)\right) \\
& =\left(\mu^{\prime}\left(r^{\prime} c^{\prime}\right) \cdot e^{\prime},\left(\vartheta^{\prime}\left(e^{\prime}\right) \cdot r^{\prime}\right) \cdot c^{\prime}, h\left(f_{2}\left(e^{\prime}\right), f_{3}^{\prime}\left(r^{\prime} c^{\prime}\right)\right)\right) \\
& =\left(\mu^{\prime}\left(r^{\prime} \cdot c^{\prime}\right) \cdot e^{\prime}, \vartheta^{\prime}\left(e^{\prime}\right) \cdot\left(r^{\prime} \cdot c^{\prime}\right), h\left(f_{2}\left(e^{\prime}\right), f_{3}^{\prime}\left(r^{\prime} \cdot c^{\prime}\right)\right)\right) \\
& =h^{\prime}\left(e^{\prime}, r^{\prime} \cdot c^{\prime}\right)
\end{aligned}
$$

7. For $c^{\prime} \in C^{\prime}, e^{\prime} \in E^{\prime}$ and $r^{\prime} \in R^{\prime}$;

$$
\begin{aligned}
r^{\prime} \cdot h^{\prime}\left(e^{\prime}, c^{\prime}\right) & =r^{\prime} \cdot\left(\mu^{\prime}\left(c^{\prime}\right) \cdot e^{\prime}, \vartheta^{\prime}\left(e^{\prime}\right) \cdot c^{\prime \prime}, h\left(f_{2}\left(e^{\prime}\right), f_{3}^{\prime}\left(c^{\prime}\right)\right)\right) \\
& =r^{\prime} \cdot\left(\mu^{\prime}\left(c^{\prime}\right) \cdot e^{\prime}\right), r^{\prime} \cdot\left(\vartheta^{\prime}\left(e^{\prime}\right) \cdot c^{\prime \prime}\right), r^{\prime} \cdot h\left(f_{2}\left(e^{\prime}\right), f_{3}^{\prime}\left(c^{\prime}\right)\right) \\
& =\left(r^{\prime} \cdot \mu^{\prime}\left(c^{\prime}\right)\right) \cdot e^{\prime},\left(r^{\prime} \cdot \vartheta^{\prime}\left(e^{\prime}\right)\right) \cdot c^{\prime \prime}, h\left(r^{\prime} \cdot f_{2}\left(e^{\prime}\right), f_{3}^{\prime}\left(c^{\prime}\right)\right) \\
& =\left(\mu^{\prime}\left(c^{\prime}\right) \cdot r^{\prime} \cdot e^{\prime}, \vartheta^{\prime}\left(r^{\prime} e^{\prime}\right) \cdot c^{\prime \prime}, h\left(f_{2}\left(r^{\prime} \cdot e^{\prime}\right), f_{3}^{\prime}\left(c^{\prime}\right)\right)\right) \\
& =\left(\mu^{\prime}\left(c^{\prime}\right) \cdot\left(r^{\prime} \cdot e^{\prime}\right), \vartheta^{\prime}\left(r^{\prime} \cdot e^{\prime}\right) \cdot c^{\prime \prime}, h\left(f_{2}\left(r^{\prime} \cdot e^{\prime}\right), f_{3}^{\prime}\left(c^{\prime}\right)\right)\right) \\
& =h^{\prime}\left(r^{\prime} \cdot e^{\prime}, c^{\prime}\right) \\
r^{\prime} \cdot h^{\prime}\left(e^{\prime}, c^{\prime}\right) & =r^{\prime} \cdot\left(\mu^{\prime}\left(c^{\prime}\right) \cdot e^{\prime}, \vartheta^{\prime}\left(e^{\prime}\right) \cdot c^{\prime \prime}, h\left(f_{2}\left(e^{\prime}\right), f_{3}^{\prime}\left(c^{\prime}\right)\right)\right) \\
& =r^{\prime} \cdot\left(\mu^{\prime}\left(c^{\prime}\right) \cdot e^{\prime}\right), r^{\prime} \cdot\left(\vartheta^{\prime}\left(e^{\prime}\right) \cdot c^{\prime \prime}\right), r^{\prime} \cdot h\left(f_{2}\left(e^{\prime}\right), f_{3}^{\prime}\left(c^{\prime}\right)\right) \\
& =\left(r^{\prime} \cdot \mu^{\prime}\left(c^{\prime}\right)\right) \cdot e^{\prime},\left(r^{\prime} \cdot \vartheta^{\prime}\left(e^{\prime}\right)\right) \cdot c^{\prime \prime}, h\left(r^{\prime} \cdot f_{2}\left(e^{\prime}\right), f_{3}^{\prime}\left(c^{\prime}\right)\right) \\
& =\left(\mu^{\prime}\left(r^{\prime} c^{\prime}\right) \cdot e^{\prime},\left(\vartheta^{\prime}\left(e^{\prime}\right) \cdot r^{\prime}\right) \cdot c^{\prime}, h\left(f_{2}\left(e^{\prime}\right), f_{3}^{\prime}\left(r^{\prime} c^{\prime}\right)\right)\right) \\
& =\left(\mu^{\prime}\left(r^{\prime} \cdot c^{\prime}\right) \cdot e^{\prime}, \vartheta^{\prime}\left(e^{\prime}\right) \cdot\left(r^{\prime} \cdot c^{\prime}\right), h\left(f_{2}\left(e^{\prime}\right), f_{3}^{\prime}\left(r^{\prime} \cdot c^{\prime}\right)\right)\right) \\
& =h^{\prime}\left(e^{\prime}, r^{\prime} \cdot c^{\prime}\right)
\end{aligned}
$$

8. For $c^{\prime} \in C^{\prime}, e^{\prime} \in E^{\prime}$ and $r^{\prime} \in R^{\prime}$;

$$
\begin{aligned}
r^{\prime} \cdot h^{\prime}\left(e^{\prime}, c^{\prime}\right) & =r^{\prime} \cdot\left(\mu^{\prime}\left(c^{\prime}\right) \cdot e^{\prime}, \vartheta^{\prime}\left(e^{\prime}\right) \cdot c^{\prime \prime}, h\left(f_{2}\left(e^{\prime}\right), f_{3}^{\prime}\left(c^{\prime}\right)\right)\right) \\
& =r^{\prime} \cdot\left(\mu^{\prime}\left(c^{\prime}\right) \cdot e^{\prime}\right), r^{\prime} \cdot\left(\vartheta^{\prime}\left(e^{\prime}\right) \cdot c^{\prime \prime}\right), r^{\prime} \cdot h\left(f_{2}\left(e^{\prime}\right), f_{3}^{\prime}\left(c^{\prime}\right)\right) \\
& =\left(r^{\prime} \cdot \mu^{\prime}\left(c^{\prime}\right)\right) \cdot e^{\prime},\left(r^{\prime} \cdot \vartheta^{\prime}\left(e^{\prime}\right)\right) \cdot c^{\prime \prime}, h\left(r^{\prime} \cdot f_{2}\left(e^{\prime}\right), f_{3}^{\prime}\left(c^{\prime}\right)\right) \\
& =\left(\mu^{\prime}\left(c^{\prime}\right) \cdot r^{\prime} \cdot e^{\prime}, \vartheta^{\prime}\left(r^{\prime} e^{\prime}\right) \cdot c^{\prime \prime}, h\left(f_{2}\left(r^{\prime} \cdot e^{\prime}\right), f_{3}^{\prime}\left(c^{\prime}\right)\right)\right) \\
& =\left(\mu^{\prime}\left(c^{\prime}\right) \cdot\left(r^{\prime} \cdot e^{\prime}\right), \vartheta^{\prime}\left(r^{\prime} \cdot e^{\prime}\right) \cdot c^{\prime \prime}, h\left(f_{2}\left(r^{\prime} \cdot e^{\prime}\right), f_{3}^{\prime}\left(c^{\prime}\right)\right)\right) \\
& =h^{\prime}\left(r^{\prime} \cdot e^{\prime}, c^{\prime}\right)
\end{aligned}
$$




$$
\begin{aligned}
r^{\prime} \cdot h^{\prime}\left(e^{\prime}, c^{\prime}\right) & =r^{\prime} \cdot\left(\mu^{\prime}\left(c^{\prime}\right) \cdot e^{\prime}, \vartheta^{\prime}\left(e^{\prime}\right) \cdot c^{\prime \prime}, h\left(f_{2}\left(e^{\prime}\right), f_{3}^{\prime}\left(c^{\prime}\right)\right)\right) \\
& =r^{\prime} \cdot\left(\mu^{\prime}\left(c^{\prime}\right) \cdot e^{\prime}\right), r^{\prime} \cdot\left(\vartheta^{\prime}\left(e^{\prime}\right) \cdot c^{\prime \prime}\right), r^{\prime} \cdot h\left(f_{2}\left(e^{\prime}\right), f_{3}^{\prime}\left(c^{\prime}\right)\right) \\
& =\left(r^{\prime} \cdot \mu^{\prime}\left(c^{\prime}\right)\right) \cdot e^{\prime},\left(r^{\prime} \cdot \vartheta^{\prime}\left(e^{\prime}\right)\right) \cdot c^{\prime \prime}, h\left(r^{\prime} \cdot f_{2}\left(e^{\prime}\right), f_{3}^{\prime}\left(c^{\prime}\right)\right) \\
& =\left(\mu^{\prime}\left(r^{\prime} c^{\prime}\right) \cdot e^{\prime},\left(\vartheta^{\prime}\left(e^{\prime}\right) \cdot r^{\prime}\right) \cdot c^{\prime}, h\left(f_{2}\left(e^{\prime}\right), f_{3}^{\prime}\left(r^{\prime} c^{\prime}\right)\right)\right) \\
& =\left(\mu^{\prime}\left(r^{\prime} \cdot c^{\prime}\right) \cdot e^{\prime}, \vartheta^{\prime}\left(e^{\prime}\right) \cdot\left(r^{\prime} \cdot c^{\prime}\right), h\left(f_{2}\left(e^{\prime}\right), f_{3}^{\prime}\left(r^{\prime} \cdot c^{\prime}\right)\right)\right) \\
& =h^{\prime}\left(e^{\prime}, r^{\prime} \cdot c^{\prime}\right)
\end{aligned}
$$

9. For $\left(e^{\prime}, c^{\prime}, d\right) \in f^{*}$ and $e^{\prime \prime} \in E^{\prime}$;

$$
\begin{aligned}
h^{\prime}\left(e^{\prime}, \beta_{1}\left(e^{\prime}, c^{\prime}, d\right)\right) & =h^{\prime}\left(e^{\prime \prime}, c^{\prime}\right) \\
& =\left(\mu \prime\left(c^{\prime}\right) \cdot e^{\prime \prime}, \vartheta^{\prime}\left(e^{\prime \prime}\right) \cdot c^{\prime}, h\left(f_{2}\left(e^{\prime \prime}\right), f^{\prime}\left(c^{\prime}\right)\right)\right) \\
& =\vartheta^{\prime}\left(e^{\prime}\right) \cdot e^{\prime \prime}, \vartheta^{\prime}\left(e^{\prime \prime}\right) \cdot c^{\prime}, h\left(f_{2}\left(e^{\prime \prime}\right), \sigma(d)\right) \\
& =\left(e^{\prime} \cdot e^{\prime \prime}, e^{\prime \prime} \cdot c^{\prime}, f_{2}\left(e^{\prime \prime}\right) \cdot d\right) \\
& =e^{\prime \prime} \cdot\left(e^{\prime}, c^{\prime}, d\right)
\end{aligned}
$$

10. For $\left(e^{\prime}, c^{\prime}, d\right) \in f^{*}$ and $c^{\prime \prime} \in C^{\prime}$;

$$
\begin{aligned}
h^{\prime}\left(\beta_{2}\left(e^{\prime}, c^{\prime}, d\right), c^{\prime \prime}\right) & =h^{\prime}\left(e^{\prime}, c^{\prime}\right) \\
& =\left(\mu \prime\left(c^{\prime \prime}\right) \cdot e^{\prime}, \vartheta^{\prime}\left(e^{\prime}\right) \cdot c^{\prime \prime}, h\left(f_{2}\left(e^{\prime}\right), f_{3}^{\prime}\left(c^{\prime \prime}\right)\right)\right) \\
& =\left(c^{\prime \prime} \cdot e^{\prime}, \mu \prime\left(c^{\prime}\right) \cdot c^{\prime \prime}, h\left(\delta(d), f_{3}^{\prime}\left(c^{\prime \prime}\right)\right)\right) \\
& =\left(c^{\prime \prime} \cdot e^{\prime}, c^{\prime} \cdot c^{\prime \prime}, f_{3}\left(c^{\prime \prime}\right) \cdot d\right) \\
& =c^{\prime \prime} \cdot\left(e^{\prime}, c^{\prime}, d\right)
\end{aligned}
$$

Conclusion 3.3. There exist a functor

$$
F: X M o d^{2} \rightarrow \mathrm{Crs}^{2}
$$

from the category of pairs of crossed modules to that of crossed squares.

\section{Pullback in $C r s^{2}$}

In this section, we will give the construction of a pullback object in $\mathrm{Crs}^{2}$ using pairs of crossed modules.

Definition 4.1. Given a crossed square $\left(\begin{array}{ccc} & C & \\ D & & R \\ & E & \end{array}\right)$ and a morphism of pairs of crossed modules $f=\left(f_{1}, f_{2}, f_{3}\right):\left(C^{\prime}, E^{\prime}, R^{\prime}, \mu^{\prime}, \vartheta^{\prime}\right) \rightarrow(C, E, R, \mu, \vartheta)$ the pullback crossed square can be given by

$$
\begin{aligned}
& \text { i) A crossed square }\left(\begin{array}{ccc}
f^{*} & & C^{\prime} \\
& E^{\prime} & R^{\prime}
\end{array}\right) \text { such that } \\
& \qquad f^{*}=\left\{\left(e^{\prime}, c^{\prime}, d\right) \in E^{\prime} \times C^{\prime} \times D: \vartheta^{\prime}\left(e^{\prime}\right)=\mu^{\prime}\left(c^{\prime}\right), f_{2}\left(e^{\prime}\right)=\alpha(d), f_{3}\left(c^{\prime}\right)=\beta(d)\right\}
\end{aligned}
$$

ii) Given any morphism of crossed squares

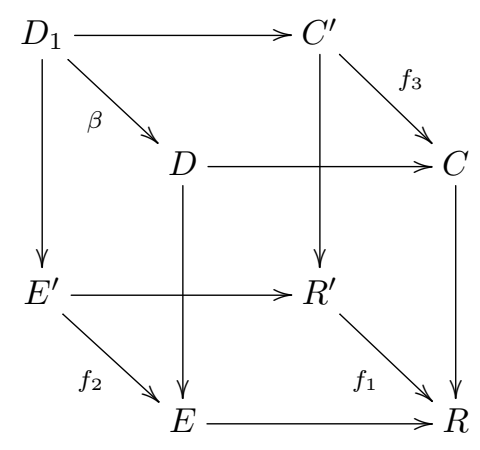


there is a unique crossed square morphism making the diagram

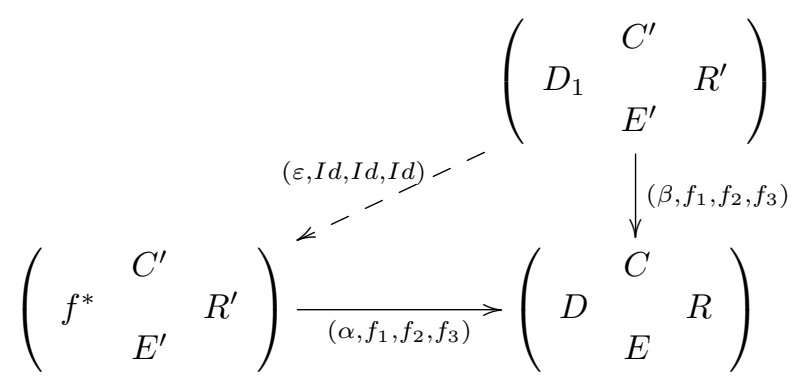

commutative.

Next we define a morphism

$$
\begin{array}{ccc}
\alpha: f^{*} & \rightarrow & D \\
\left(e^{\prime}, c^{\prime}, d\right) & \mapsto & d
\end{array}
$$

to give the following proposition.

Proposition 4.2. $\left(f^{*}, \beta_{1}, \beta_{2}\right)$ is the pullback object for the pair $\left(\mu^{\prime}, \vartheta^{\prime}\right)$.

Proof. For $\left(e^{\prime}, c^{\prime}, d\right) \in E^{\prime} \times C^{\prime} \times D$;

$$
\begin{aligned}
f_{2} \beta_{2}\left(e^{\prime}, c^{\prime}, d\right) & =f_{2}\left(e^{\prime}\right) \\
& =\delta(d) \\
& =\delta \alpha\left(e^{\prime}, c^{\prime}, d\right) \\
& \Rightarrow f_{2} \beta_{2}=\delta \alpha \\
\mu^{\prime} \beta_{1}\left(e^{\prime}, c^{\prime}, d\right) & =\mu^{\prime}\left(c^{\prime}\right) \\
& =\vartheta^{\prime}\left(e^{\prime}\right) \\
& =\vartheta^{\prime} \beta_{2}\left(e^{\prime}, c^{\prime}, d\right) \\
& \Rightarrow \mu^{\prime} \beta_{1}=\vartheta^{\prime} \beta_{2} \\
& \\
f_{3} \beta_{1}\left(e^{\prime}, c^{\prime}, d\right) & =f_{3}\left(c^{\prime}\right) \\
& =\partial(d) \\
& =\partial \alpha\left(e^{\prime}, c^{\prime}, d\right) \\
& \Rightarrow f_{3} \beta_{1}=\partial \alpha
\end{aligned}
$$

Furthermore since $\left(f_{1}, f_{2}, f_{3}\right) \in \operatorname{Mor}\left(X M o d^{2}\right)$ we have $f_{1} \mu^{\prime}=\mu f_{3}, \vartheta f_{2}=f_{1} \vartheta^{\prime}$ and $\left(\begin{array}{ccc}C & \\ D & & R \\ & E\end{array}\right)$ is a crossed square $\mu \partial=\vartheta \delta$ making the following diagram

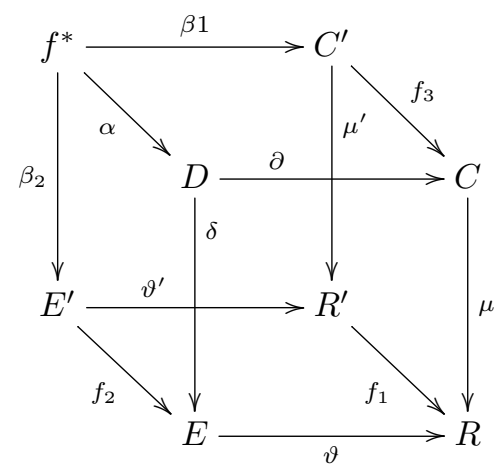


commutative. Thus $\left(\alpha, f_{1}, f_{2}, f_{3}\right)$ is a morphism in $\mathrm{Crs}^{2}$. Let $g=\left(\alpha^{\prime}, f_{1}, f_{2}, f_{3}\right)$ be another morphism in $C r s^{2}$

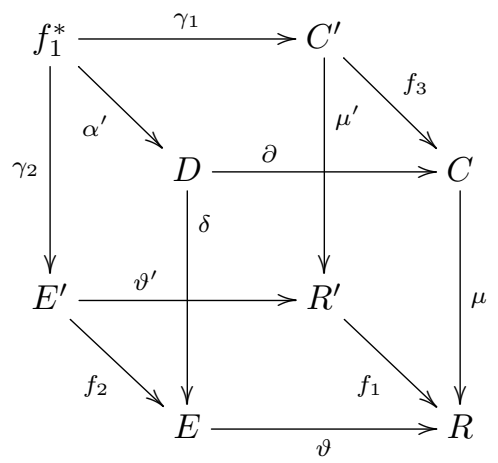

and an h-map

$$
\begin{array}{ccc}
h_{1}: E^{\prime} \times C^{\prime} & \rightarrow & f_{1}^{*} \\
\left(e^{\prime}, c^{\prime}\right) & \mapsto & x
\end{array}
$$

be given. Define

$$
\begin{aligned}
\xi: f_{1}^{*} & \rightarrow f^{*} \\
x & \mapsto\left(\gamma_{1}(x), \gamma_{2}(x), \alpha^{\prime}(x)\right)
\end{aligned}
$$

Next we will show that $\xi: f_{1}^{*} \rightarrow f^{*}$ is well-defined. We know that for

$$
\left(e^{\prime}, c^{\prime}, d\right) \in f^{*} \Leftrightarrow \vartheta^{\prime}\left(e^{\prime}\right)=\mu^{\prime}\left(c^{\prime}\right), f_{3}\left(c^{\prime}\right)=\partial(d), f_{2}\left(e^{\prime}\right)=\delta(d)
$$

For $x \in f_{1}^{*}$

$$
\begin{aligned}
& f_{3}\left(\gamma_{2}(x)\right)=f_{3} \gamma_{2}(x)=\partial \alpha^{\prime}(x)=\partial\left(\alpha^{\prime}(x)\right) \\
& f_{2}\left(\gamma_{1}(x)\right)=f_{2} \gamma_{1}(x)=\delta \alpha^{\prime}(x)=\delta\left(\alpha^{\prime}(x)\right)
\end{aligned}
$$

This shows that $\left(\gamma_{1}(x), \gamma_{2}(x), \alpha^{\prime}(x)\right) \in f^{*}$. Then we get

$$
\begin{aligned}
& \alpha \xi(x)=\alpha\left(\gamma_{1}(x), \gamma_{2}(x), \alpha^{\prime}(x)\right)=\alpha^{\prime}(x) \\
& \beta_{1} \xi(x)=\beta_{1}\left(\gamma_{1}(x), \gamma_{2}(x), \alpha^{\prime}(x)\right)=\gamma_{1}(x) \\
& \beta_{2} \xi(x)=\beta_{2}\left(\gamma_{1}(x), \gamma_{2}(x), \alpha^{\prime}(x)\right)=\gamma_{2}(x)
\end{aligned}
$$

That is we have the following diagram

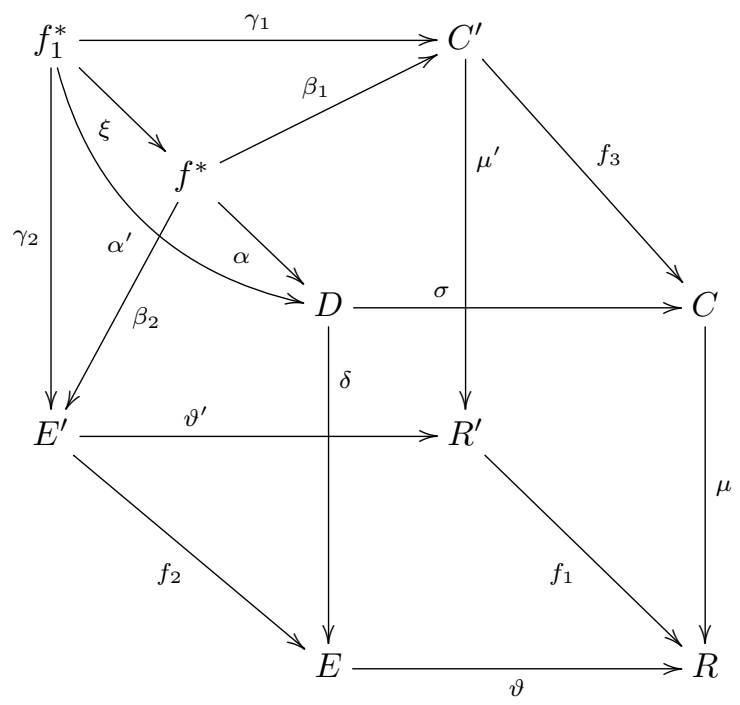

Let

$$
\begin{aligned}
\xi^{\prime}: f_{1}^{*} & \rightarrow f^{*} \\
x & \mapsto\left(e^{\prime}, c^{\prime}, d\right)
\end{aligned}
$$


be another morphism satisfying

$$
\beta_{1} \xi^{\prime}(x)=\gamma_{1}(x), \beta_{2} \xi^{\prime}(x)=\gamma_{2}(x), \alpha \xi^{\prime}(x)=\alpha^{\prime}(x) .
$$

For $x \in f_{1}^{*}$ we have;

$$
\begin{aligned}
& \beta_{1} \xi^{\prime}(x)=\beta_{1}\left(e^{\prime}, c^{\prime}, d\right)=c^{\prime}=\gamma_{1}(x) \\
& \beta_{2} \xi^{\prime}(x)=\beta_{2}\left(e^{\prime}, c^{\prime}, d\right)=e^{\prime}=\gamma_{2}(x) \\
& \alpha \xi^{\prime}(x)=\alpha\left(e^{\prime}, c^{\prime}, d\right)=d=\alpha^{\prime}(x)
\end{aligned}
$$

this implies $\left(\gamma_{1}(x), \gamma_{2}(x), \alpha^{\prime}(x)\right)=\left(e^{\prime}, c^{\prime}, d\right)$. Since

$$
\begin{aligned}
\xi^{\prime}(x) & =\left(e^{\prime}, c^{\prime}, d\right) \\
& =\left(\gamma_{1}(x), \gamma_{2}(x), \alpha^{\prime}(x)\right) \\
& =\xi(x) \\
& \Rightarrow \xi^{\prime}(x)=\xi(x) \\
& \Rightarrow \xi^{\prime}=\xi
\end{aligned}
$$

$\xi$ is unique. As a result $\left(f^{*}, \beta_{1}, \beta_{2}\right)$ is the pullback object for the pair $\left(\mu^{\prime}, \vartheta^{\prime}\right)$.

Conclusion 4.3. Pullback object exists in $\mathrm{Crs}^{2}$.

\section{References}

[1] Whitehead, J.H.C.: Combinatorial homotopy II. Bull. Amer. Math. Soc. 55, 453-496 (1949) .

[2] Ellis G.: Higher dimensional crossed module of algebras. Journal of Pure and Applied Algebra. 52, 277-282 (1988).

[3] Arvasi Z:: Crossed Squares and 2 Crossed Modules of Commutative Algebras. Theory and Applications of Categories. 3 (7), 160-181 (1997).

[4] Arvasi Z., Porter, T.: Higher-dimensional Peiffer elementsin simplicial Commutative Algebras. Theory and Applications of Categories. 3 (1), 1-23 (1997).

[5] Arvasi Z., Porter T.: Simplicial and Crossed Resolutions of Commutative Algebras. Journal of Algebra. 181, 426-448 (1996).

[6] Brown, R.,Higgins, P.: On the Connection between the Second Relative Homotopy Groups of some Related Spaces. Proc. London Math. Soc. 36(2), 193-212 (1978).

[7] Brown, R., Sivera. R.: Algebraic colimit calculations in homotopy theory using fibred and cofibred categories. Theory and Application of Categories. 22(8), 222-251 (2009).

[8] Porter, T.: Homology of commutative algebras and an invariant of Simis and Vasconcelos. Journal of Algebra. 99, 458-465 (1986).

[9] Gerstenhaber, M.: On the deformation of rings and algebras. Annual of Mathematics. 84, 1-19 (1966).

[10] Lichtenbaum, S., Schlessinger, M.: The cotangent complex of a morphism. Transection American Matematics Society. 128, 41-70 (1967).

[11] Guin-Waléry, D., Loday, J-L.: Obstructioná l'excision en K-théorie algébrique, in: Algebraic K-Theory (Evanston 1980). Lecture Notes in Math. 854, 179-216 (1981).

[12] Conduché, D.: Modules croisés généralisés de longueur 2. Journal of Pure and Applied Algebra. 34, 155-178 (1984).

[13] Arvasi, Z., Ulualan, E.: Quadratic and 2-crossed modules of algebras. Algebra Colloquium. 14, 669-686 (2007).

[14] Grandjéan, A.R., Vale, M,J.: 2-Modulos cruzados an la cohomologia de André- Quillen. Memorias de la Real Academia de Ciencias. 22, 1-28 (1986).

[15] Shammu, N.M.: Algebraic and categorical structure of categories of crossed modules of algebras. Ph.D. thesis. University College of North Wales (1992).

[16] Arslan, U. E., Akca, I., Irmak, G. O., Avcio ğlu, O.: Fibrations of 2-crossed modules. Mathematical Methods in the Applied Sciences. 42(16), 5293-5304 (2018). 


\section{Affiliations}

ELIS SOYLU YILMAZ

AdDRESS: Eskişehir Osmangazi University, Dept. of Mathematics - Computer Science , 26040, Eskişehir- Turkey. E-MAIL: esoylu@ogu.edu.tr

ORCID ID:http://orcid.org/0000-0002-0869-310x

KORAY YILMAZ

AdDress: Kütahya Dumlupınar University, Dept. of Mathematics, 43000, Kütahya-Turkey.

E-MAIL: koray.yilmaz@dpu.edu.tr

ORCID ID:https://orcid.org/0000-0002-8641-0603 